\title{
Covid-19: an Imperative to Bridge the Gap Between Medicine And Public Health
}

\author{
Alvin I. Mushlin, MD, SCM \\ $\mathrm{J}$ Gen Intern Med 35(8):2445-6 \\ DOI: $10.1007 / \mathrm{s} 11606-020-05932-\mathrm{w}$ \\ (c) Society of General Internal Medicine 2020
}

$\mathrm{T}$ he gap between public health and medical care has not served us well. The current pandemic of the disease caused by SARS-CoV-2 tragically paints this picture only too clearly. We may have been able to avert this crisis, and certainly would have been better prepared to deal with it, if these two elements of our health care systems had been working smoothly and efficiently as partners.

Medicine and public health share a common mission, the minimization of disease and the alleviation of human suffering. However, important differences have prevented their integration and effective working collaboration (see Table 1) both presently and in the past. ${ }^{1}$

While medicine is essentially aimed at the diagnosis and treatment of diseases, the role of public health is primarily the prevention of disease. In medicine, reasoning derives from a fundamental understanding of disease biology and pathophysiology, and the scientific rationale for therapeutics, whereas decisions in public health result from probabilistic inferences based on counts and statistics of diseases in populations.

Much has been written about this divide and its consequences, and multiple attempts have been made to create a unified infrastructure. ${ }^{2}$ However, these efforts have been thwarted by separation of the public and private sectors, their funding, and the responsibilities assumed by each, as well as by differences in education and licensure of professionals and a history and culture that assumes that medicine must advocate for individuals as opposed to the "medical commons.",3

In the past, there was less urgency to resolve these differences because almost everything needed to prevent major diseases could be accomplished through the public health infrastructure alone. For example, cholera could be prevented through sanitation of the water supply, tuberculosis via proper ventilation and adequate housing, typhoid fever through clean food inspections and handling, tropical diseases via control of

Received April 24, 2020

Accepted May 18, 2020

Published online June 3, 2020 insect vectors, and common infectious diseases through immunizations.

However, now we are left with a different spectrum of illnesses that are not solvable by either discipline working alone. Presently, the major causes of death and disability are chronic illnesses, including heart disease and cancer, and modern global infectious diseases such as Covid-19.

Chronic illnesses are largely the result of genetic factors, often pared with difficult to alter life style aberrations. Their control requires medical technologies for screening and early diagnosis, frequently life-long treatments, and anticipatory medical care for the prevention of complications. Public health is needed for population-based initiatives to facilitate screening and medical care, to foster healthy lifestyle choices, and to eliminate environmental disease factors.

Modern global infectious diseases, as we are now seeing with the emergence of Covid-19, require an even more robust partnership. The medical care system must provide lifesaving treatment and be relied on for testing and early identification of cases. Just as importantly, it must also engage with individuals to encourage preventive measures. Flexner envisioned this over a century ago when he wrote: “The physician's relationship was formerly to his patient - at most to his patient's family - and it was almost altogether remedial. If the patient had something the matter with him; the doctor was called in to cure it........But the physician's function is fast becoming social and preventive, rather than individual and curative. Upon him society relies to ascertain, and ( through measures essentially educational) to enforce, the conditions that prevent disease and make positively for physical and moral well-being." "4 This seems especially prescient now during Covid-19.

However, it's clear that medicine can't deal with epidemics alone. Disease containment requires public health surveillance and early recognition of index cases, then meticulous contact tracing and quarantine when necessary. Mitigating and slowing the spread of the disease demands community interventions and education to implement preventive measures such as hand washing and social distancing. So as not to overwhelm the health care system, we need to create guidelines and standards, educate people, and provide planning for and distribution of supplies such as diagnostic tests, respirators, and personal protective equipment. As we see during the current crisis, public health and government efforts are also required to augment and support health professionals. It is 
Table 1 A Brief Summary of Differences Between Medicine And Public Health (Adapted From ${ }^{1}$ )

Characteristics of Medicine

Purpose is cure of disease and alleviation of suffering in individuals Emphasis on treatment of illness

Based largely on basic biology and biomedical sciences

Uses deterministic reasoning based on pathophysiology

Located in hospitals, clinics, and offices

Characteristics of Public health

Goal is the prevention or control of disease in a population

Emphasis on disease prevention and early detection

Depends on epidemiology and statistics

Relies on probabilistic analysis and causal inference

Based in organizations, mostly within governments and communities

sometimes necessary to license additional personnel and expeditiously approve and introduce new diagnostic tests and treatments.

A strong alliance between the two fields is also required to inform the steps necessary to control the epidemic. The development of diagnostic tests and evaluating their accuracy can only be accomplished when studied in those with and without the disease in a representative overall population. Such population-based studies are also the most valid way to observe and document the characteristics of patients versus normal controls and the clinical course of patients with the disease so that the most discriminatory symptoms and signs, and prognostic features can be identified. This information then becomes the evidence foundation for timely diagnosis and prognosis, and the basis to inform the public about when to seek medical care. Community-based epidemiologic studies using validated tests can then quantify the true disease incidence and prevalence, and the presence of antibodies against the virus in both symptomatic and asymptomatic individuals. With this information, individuals can be informed about the average overall risk of disease, the risk in people like them, and the impact of the disease on society can be estimated. In order to target preventive interventions effectively, casecontrol studies are needed to understand those at most risk, including demographic, clinical and immunological characteristics, as well as behavioral, environmental, and community factors. So far, these data have been scarce in this epidemic.
Thus, although an understanding of the biology of the virus and the disease is, of course, critically needed, the generation of knowledge outlined in the previous paragraph through these types of "shoe leather epidemiology" studies to identify the causes of the virus's emergence and spread, and to inform and develop clinical and preventive strategies, is the linchpin for controlling the epidemic and for preventing its recurrence.

If this integration had been accomplished before Covid-19 struck, and a balanced agenda for action and research had emerged as a result, it would have been possible to contain this epidemic early on. At the least, it would have been less threatening than it has become. The control of the epidemic in Germany and New Zealand was largely because of such integration. The spread of the virus and its consequences in major cities such as Seoul and Hong Kong were likely spared because of it. Narrowing the divide between public health and medicine is needed now more than ever before.

Corresponding Author: Alvin I. Mushlin, MD, ScM; Departments of Population Health Sciences and Medicine, Weill Cornell Medicine, New York, NY, USA (e-mail: aim2001@med.cornell.edu).

\section{Compliance with Ethical Standards:}

Conflict of Interest: The author (Alvin I Mushlin) reports no conflicts of interest related to this submission.

\section{REFERENCES}

1. Fineberg, H.V., Public health and medicine where: the twain shall meet. Am J Prev Med, 2011. 41(4 Suppl 3): p. S149-51.

2. Beitsch, L.M., et al., The medicine and public health initiative ten years later. Am J Prev Med, 2005. 29(2): p. 149-53.

3. Hiatt, H.H., Protecting the medical commons: who is responsible? N Engl J Med, 1975. 293(5): p. 235-41.

4. Flexner, A., Medical education in the United States and Canada. From the Carnegie Foundation for the Advancement of Teaching, Bulletin Number Four, 1910. Bull World Health Organ, 2002. 80(7): p. 594-602.

Publisher's Note: Springer Nature remains neutral with regard to jurisdictional claims in published maps and institutional affiliations. 